\title{
Urgences médicales au cabinet dentaire : une enquête française
}

\section{Medical emergencies in dental practice: a french survey}

\author{
BARTHOLOMEUS CALON*, ALICE BILDSTEIN**, JESSICA SAMIN* ${ }^{\star}$ ALAIN MEYER*, \\ OLIVIER ATTARD*, JEAN RIEGER ${ }^{\star \star}$, ABDESSAMAD BOUKARI $^{\star \star}$, AHMED FÉKI ** $^{*}$
}

\begin{abstract}
RÉSUMÉ
Objectif : Bien que rares, les urgences médicales survenant dans un cabinet dentaire ne doivent pas être méconnues. Cette enquête a été réalisée afin d'évaluer la fréquence de survenue et le devenir des urgences vitales dans la pratique dentaire en France.

Méthode : Cette enquête a été menée entre octobre 2004 et novembre 2005 auprès d'un échantillon de 711 chirurgiens-dentistes exerçant en France (sur un total de 34 563).

Résultats : Le taux de réponse a été de $99 \%, 704$ sur 711 chirurgiens-dentistes cumulant 15223 années de pratique odontologique (21,6 années en moyenne) ont répondu à ce questionnaire. Vingt praticiens avaient dû pratiquer une réanimation cardiopulmonaire $(R C P)$ au cabinet au moins une fois au cours de leur carrière (1/35 praticiens ou 1/20 carrières actives). Paradoxalement, l'incidence des RCP était plus élevée en dehors du cabinet dentaire (27 RCP, c'est-à-dire $1 / 26$ praticiens). Environ un quart $(1 / 4,5)$ des praticiens avait fait appel au SAMU (c'est-à-dire 1/2,4 carrières). En ce qui concerne le niveau d'équipement du cabinet, $77 \%$ des praticiens disposaient d'une source d'oxygène et $40 \%$ d'un sphygmomanomètre.

Conclusions : Bien que l'incidence des urgences vitales soit rare au cabinet dentaire, $5 \%$ des praticiens devront pratiquer au moins une fois une RCP lors de leur carrière, avec succès dans $75 \%$ des cas. Lors d'une carrière active, près d'un praticien sur deux fera appel au moins une fois au SAMU. Ces données permettent de justifier l'obligation de formation des chirurgiens-dentistes aux gestes et soins d'urgence (Arrêté du 3 mars 2006), d'autant plus qu'ils y sont également confrontés en dehors du cabinet dentaire. Med Buccale Chir Buccale 2007; 13: 31-35).
\end{abstract}

mots clés: enquête santé, urgences, réanimation cardiopulmonaire, cabinet dentaire

\section{SUMMARY}

Objective. Although uncommon, medical emergencies (ME) do occur in dental practice. This survey was carried out to evaluate the occurrence and outcome of life threatening emergencies in general dental practice in France. Methods. From October 2004 to November 2005, 711 general dental practitioners (GDP) exercising in France were surveyed concerning the occurrence of ME during their active career.

Results. The response rate was 99\% (704/711 GDP's, mean practice time: 21,6 years). Twenty (20) GDP allegedly had performed cardiopulmonary resuscitation (CPR) in the dental office at least once in their career, affecting one in thirty five (1/35) GDP, or one in twenty (1/20) practising lifetimes (40 years). Remarkably, even more GDP (27, i.e.1/26) had performed CPR outside the dental office, during non professional activities. Vasovagal syncope in

\footnotetext{
* Service d'Anesthésie - Réanimation Chirurgicale, Hôpitaux Universitaires de Strasbourg, CHU de Hautepierre

** Département de Médecine Buccale et Chirurgie Buccale, Faculté de Chirurgie Dentaire de Strasbourg

Demande de tirés à part:

Bartholomeus Calon, Service de Réanimation Chirurgicale 6361, CHU de Hautepierre, Avenue Molière

67098 Strasbourg Cedex bartholomeus.calon@chru-strasbourg.fr
} 
médecine

buccale

chirurgie

buccale

VOL. $13, \mathrm{~N}^{\circ} 1$ 2007

page 32

dental practice was 6 times as frequent as other non life threatening conditions. Approximately one in four (1/4.5) GDP had ever called the emergency medical services (EMS: 1/2.4 practising lifetimes). Concerning the state of preparedness of the dental office, 77\% of all GDP had an oxygen source available, and 40\% a sphygmomanometer.

Conclusion. Although life threatening medical emergencies are uncommon in dental practice, 1 in 35 surveyed GDP had to cope with CPR at least once in the dental office ( and even more during extra professional activities). These data warrant regular training in the use of emergency drugs and basic life support (BLS) for dentists as health care providers. Med Buccale Chir Buccale 2007; 13: 31-35).

key words: health survey, emergencies, cardiopulmonary resuscitation, dental offices

Bien que rares, les urgences médicales survenant au cabinet dentaire existent et occasionnent quelques appels au SAMU. Toutefois, aucune donnée sur leur incidence réelle n'existe en France. Cette enquête a été réalisée afin d'évaluer la fréquence de survenue et le devenir des urgences vitales et non-vitales dans la pratique dentaire en France.

\section{METHODE}

Cette enquête sur la survenue d'urgences vitales ou de malaises divers au cours d'une carrière professionnelle a été menée entre octobre 2004 et novembre 2005 auprès d'un échantillon de 711 chirurgiens-dentistes exerçant en France (sur un total de 34563). Cette évaluation a été réalisée au cours de séances d'enseignement postuniversitaire. Le questionnaire comportait 9 éléments : le nombre d'années de pratique odontologique, le nombre de gestes d'urgence effectués dans l'environnement professionnel et civil, l'incidence annuelle de malaises vagaux ou autres au cabinet, le nombre d'appels à un SMUR, le nombre de décès dans les suites immédiates de soins dentaires, la présence d'une source d'oxygène et/ou d'un sphygmomanomètre au cabinet. Un questionnaire à choix multiple (QCM) était également proposé afin d'évaluer les notions médicales de base (diabète, grossesse, maladies cardio-vasculaires, etc.) de chaque praticien.

\section{RESULTATS}

Le taux de réponse a été de $99 \%, 704$ praticiens dentaires cumulant 15223 années de pratique odontologique $(21,6$ années en moyenne, minimum 1 an et maximum 40 ans) ont répondu à ce questionnaire lors de séances de formation continue. Vingt praticiens avaient dû pratiquer une réanimation cardiopulmonaire (RCP) au moins une fois au cabinet au cours de leur carrière, ce qui représente $1 / 35$ praticiens ou $\pm 1 / 20$ carrières actives (en utilisant une moyenne de 40 années pour une carrière ${ }^{[4]}$ ). Le taux de survie a été de $75 \%$. Il est à noter que l'incidence des RCP était plus élevée en dehors du cabinet dentaire (27 RCP, c'est-àdire 1/26 praticiens), lors des activités de loisirs ou de transport.

Environ un quart $(1 / 4,5)$ des praticiens avait fait appel à un SAMU (c'est-à-dire 1/2,4 carrières) (Tab.1).

L'incidence des malaises d'origine vagale était $6,4 \mathrm{x}$ plus élevée que celle des malaise d'autres étiologies (respectivement 1615 et 250). Parmi ces malaises, 8,4\% ont occasionné un appel au SAMU. Le nombre de malaises vagaux n'est pas réparti de façon homogène : 4,8\% des praticiens rapportent $22 \%$ du total des malaises observés. Le nombre de malaises vagaux ne diminue pas avec les années de pratique, sauf peut-être en fin de carrière (Fig. 1).

En ce qui concerne le niveau d'équipement des cabinets dentaires, $77 \%$ des praticiens avaient une source d'oxygène disponible, $40 \%$ possédaient un tensiomètre (Tab. 2). 
Tableau 1 : Fréquence des RCP, appels SMUR et décès immédiats, par praticien et par 40 années d'activité professionnelle Incidence of CPR, EMS calls and immediate deaths per GDP and 40 year practising lifetime

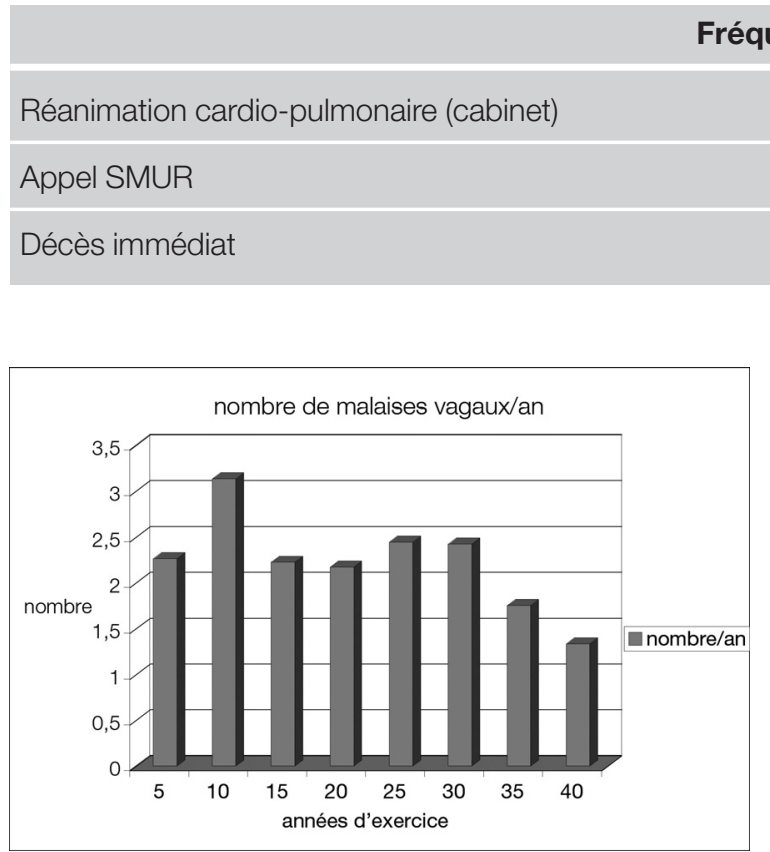

Figure 1 : Fréquence des malaises vagaux par praticien et par an, en fonction du nombre d'années d'exercice Incidence of vasovagal syncopes per GDP per year, considering the number of years of practice
Fréquence/40 ans*

$2,8 \% \quad 5,2 \%$

$22 \%$

$41 \%$

$0,7 \% \quad 1,3 \%$

* 40 ans, c'est la durée approximative de la carrière d'un chirurgien-dentiste

Tableau 2 : Niveau d'équipement du cabinet dentaire State of readiness of the dental practice

\begin{tabular}{ll}
\hline Présence d'une source d'O2 & $77 \%$ \\
\hline Présence d'un sphygmomanomètre & $40 \%$
\end{tabular}

gatoire de cet enseignement devrait limiter la portée de cet effet.

Pour l'appel téléphonique urgent, la grande majorité des dentistes fait appel au 15 pour une urgence médicale, le 112 est moins connu.

Parmi les malaises de moindre gravité, les plus fréquents (d'après la littérature) sont les syncopes vagales, les réactions aux anesthésiques locaux, l'hypoglycémie, l'angor, la crise comitale, l'inhalation bronchique et l'asthme ${ }^{[1,2,3,4]}$.

Dans notre étude, la syncope vagale était $6 \times$ plus fréquente que les autres malaises. Dans plus de 9 cas sur 10, ces syncopes n'ont pas nécessité d'appel au SAMU. II est à noter que le malaise vagal est très opérateur dépendant : moins de $5 \%$ des praticiens rapportent près d'un quart des malaises vagaux! Le nombre d'années de pratique n'a aucune influence sur l'incidence de survenue des malaises vagaux, sauf peut-être en fin de carrière. médecine

buccale chirurgie buccale

VOL. $13, \mathrm{~N}^{\circ} 1$ 2007

page 33 par voie postale. Le nombre de réponses (704) est également intéressant comparé à celui des études mentionnées (respectivement 301 , $1110,199,811)[1,2,3,4]$

II faut signaler que cette étude a été réalisée lors d'un enseignement sur les urgences médicales, ce qui peut entraîner un biais car les réponses ont été fournies par des praticiens particulièrement motivés. Toutefois le caractère dorénavant obli- 
médecine

buccale

chirurgie

buccale

VOL. $13, \mathrm{~N}^{\circ} 1$ 2007

page 34
Souvent les malaises sont imputés à une allergie aux anesthésiques locaux ( $\mathrm{AL}$ ), réaction pourtant exceptionnelle [6]. Une autre éventualité, souvent évoquée, est le surdosage en $\mathrm{AL}$ : celui-ci est également fort improbable, puisque chez l'adulte il faut l'injection de 7 carpules d'articaïne à $4 \%$ adrénalinée ou de 13 carpules de lidocaïne à $2 \%$ adrénalinée pour occasionner un surdosage [6]. En revanche, la plupart des praticiens sont conscients du risque potentiel d'allergie au latex (gants, digue).

Il faut dire ici que plusieurs appels au SAMU ont été motivés par la survenue d'un œdème de Quincke qui s'est avéré n'être qu'un emphysème sous-cutané, bénin, survenant lors de l'utilisation d'une turbine : ce cas est souvent rapporté dans la littérature, et certains ont même réalisé une biopsie pour diagnostiquer l'emphysème souscutané $[7]$.

Les malaises chez le diabétique sont rares selon la littérature $(0,17$ / praticien/an [1]). A ce propos, on doit noter que la plupart des trousses d'urgence contiennent un flacon de soluté glucosé hypertonique (a priori peu utile), mais pas de morceaux de sucre.

Plusieurs praticiens rapportent la survenue d'un glaucome aigu lors d'un geste douloureux ou lors de l'injection d'AL avec vasoconstricteur ; cet incident n'est pas mentionné dans la littérature. II ne peut s'agir que d'un glaucome à angle fermé ; il ne faut pas méconnaître cette pathologie qui, contrairement au glaucome à angle ouvert, représente une contre-indication à l'utilisation de vasoconstricteurs ${ }^{[8]}$.

En ce qui concerne les urgences vitales, il est difficile de déterminer toutes les causes des arrêts cardiorespiratoires survenus au cabinet dentaire et ayant nécessité une RCP. Leur évolution (75\% de survie) est relativement satisfaisante ; l'évolution est identique dans l'étude australienne ${ }^{[4]}$. Concernant le monitorage, $40 \%$ des cabinets dentaires disposent d'un tensiomètre, alors que l'équipement pour réaliser la mesure de la glycémie, un ECG et l'oxymétrie de pouls est exceptionnel. La réalisation de ces examens est plus fréquente dans les pays anglo-saxons probablement parce qu'ils utilisent couramment le mélange protoxyde d'azote/oxygène ; cet usage n'est pas autorisé dans les cabinets dentaires en France. L'équipement en matériel et médicaments pour la prise en charge des urgences médicales a été évalué : il existe une source d'oxygène dans $77 \%$ des cabinets dentaires. Toutefois, les contrôles techniques et les dates de péremption de la source d'oxygène ne sont guère respectés ; il semble que le service après vente de ce matériel soit très mal assuré sur le territoire français.

La législation actuelle n'autorisant pas l'acquisition d'un défibrillateur (semi-) automatique, il n'y en a pas dans les cabinets dentaires. L'évolution probable de la législation doit amener les praticiens à se familiariser d'ores et déjà avec ce matériel.

La quasi-totalité des praticiens en exercice (97\% des sujets interrogés pratiquaient depuis plus de 5 ans) n'a jamais eu d'enseignement formel de secourisme et, lorsqu'ils ont reçu une formation, c'est dans le cadre d'activités sportives ou de loisir. L'arrêté relatif à l'attestation de formation aux gestes d'urgences paru au Journal Officiel du 10 mars 2006 stipule que les professionnels de santé sont concernés par celle-ci. Pour les chirurgiens-dentistes, cette formation doit donc comprendre l'enseignement des gestes d'urgence pour les pathologies susceptibles de favoriser un arrêt cardiorespiratoire ainsi que l'adaptation du cabinet dentaire (personnel, dossier/patient, matériel et médications). Le cabinet est bien souvent adapté à la pratique de la chirurgie dentaire, mais il faut rappeler qu'il doit aussi permettre au praticien de faire face à toutes les urgences médicales, y compris celles qui ne sont pas en rapport avec l'activité professionnelle.

\section{CONCLUSION}

Bien que l'incidence des urgences vitales soit rare au cabinet dentaire, un praticien sur 20 devra pratiquer au moins une fois une RCP lors de sa carrière. Une enquête australienne, réalisée en 1997 [4], avait donné des résultats équivalents : une incidence de 7,6\% et un taux de survie de $75 \%$. Lors d'une carrière active, un praticien est confronté à 2,7 malaises d'origine diverse par an en moyenne et près d'un praticien sur deux fera 
appel au moins une fois au SAMU. Ces données permettent de justifier l'obligation de formation des chirurgiens-dentistes aux gestes et soins d'urgence (Arrêté du 3 mars 2006), d'autant plus qu'ils y sont confrontés aussi en dehors du cabinet dentaire. Le rôle du SAMU est bien sûr primordial dans la prise en charge des patients présentant une urgence médicale au cabinet dentaire, mais

\section{RÉFÉRENCES}

1 - Girdler NM, Smith DG. Prevalence of emergency events in british dental practice and emergency management skills of british dentists. Resuscitation1999; 41:15967.

2 - Atherton GJ, McCaul JA, Williams SA. Medical emergencies in general dental practice in Great Britain. Part 3: Perceptions of training and competence of GDPs in their management. Br Dent J 1999 ;186:234-7.

3 - Broadbent JM, Thomson WM. The readiness of New Zealand general dental practitioners for medical emergencies. N Z Dent J 2001 ; 97:82-6.

4 - Chapman PJ. Medical emergencies in dental practice and choice of emergency drugs and equipment : a survey of Australian dentists. Austr Dent J 1997 ; 42:103-8. également pour assurer l'enseignement, la formation et l'évaluation des chirurgiens-dentistes et de leur personnel.

Remerciements : les auteurs tiennent à remercier tous les chirurgiens-dentistes ayant participé à cette étude.
5 - Fast TB, Martin MD, Ellis TM. Emergency preparedness: a survey of dental practionners. JADA 1986 ; 112 :499501.

6 - Haas DA. An update on local anesthetics in dentistry. J Can Dent Assoc 2002 ; 68 546-51.

7 - Frühauf J, Weinke R, Pilger U, Kerl H, Müllegger RR . Soft tissue cervicofacial emphysema after dental treatment. Arch Dermatol 2005; 141 :1437-40.

8 - Madrid C. Glaucome et vasoconstricteurs. Med Buc Chir Buc 2003 ; 9 :147-8. médecine

buccale chirurgie buccale

VOL. $13, \mathrm{~N}^{\circ} 1$ 2007

page 35 